\title{
Autoimmune Lymphoproliferative Syndrome
}

National Cancer Institute

\section{Source}

National Cancer Institute. Autoimmune Lymphoproliferative Syndrome. NCI Thesaurus. Code C37864.

An autoimmune hematologic disorder characterized by autoimmune hemolytic anemia, thrombocytopenia, lymphadenopathy, hepatomegaly and splenomegaly. Patients are at an increased risk of developing Hodgkin and non-Hodgkin lymphomas. 